Supporting Information

\title{
Gold nanoparticle enhanced charge transfer in thin film assemblies of porphyrin-fullerene dyads
}

\author{
Anne Kotiaho, ${ }^{*, a}$ Riikka M. Lahtinen, ${ }^{a}$ Nikolai V. Tkachenko, ${ }^{\text {a }}$ Alexander Efimov, ${ }^{\text {a Aiko }}$ \\ Kira, ${ }^{b}$ Hiroshi Imahori ${ }^{\text {b,c }}$ and Helge Lemmetyinen ${ }^{a}$ \\ *anne.kotiaho@tut.fi \\ a Institute of Materials Chemistry, Tampere University of Technology, P.O. Box 541, 33101 \\ Tampere, Finland \\ b Department of Molecular Engineering, Graduate School of Engineering, Kyoto University, \\ Nishikyo-ku, Kyoto 615-8510, Japan \\ c Fukui Institute for Fundamental Chemistry, Kyoto University, 34-4, Takano-Nishihiraki-cho, \\ Sakyo-ku, Kyoto 606-8103, Japan
}

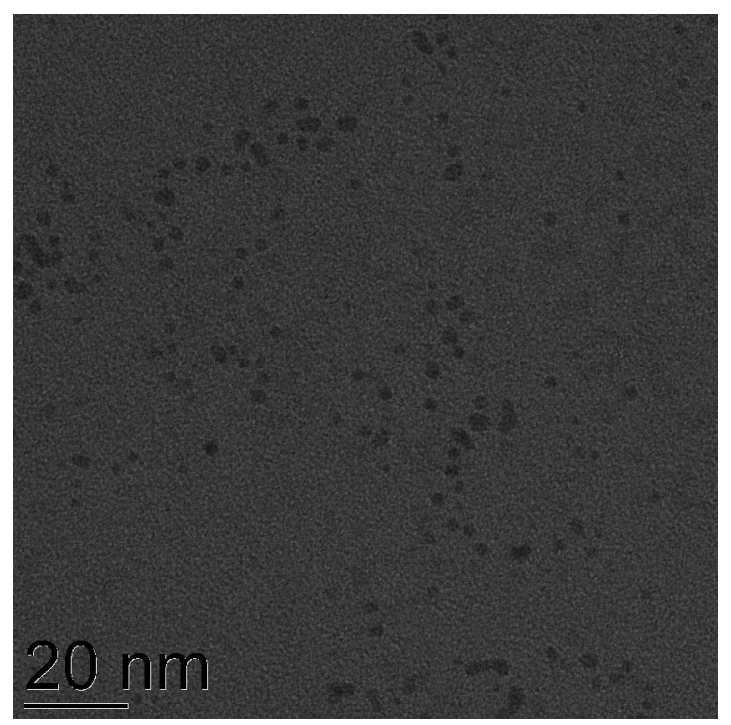

Figure S1. TEM image of the gold nanoparticles drop casted from toluene.
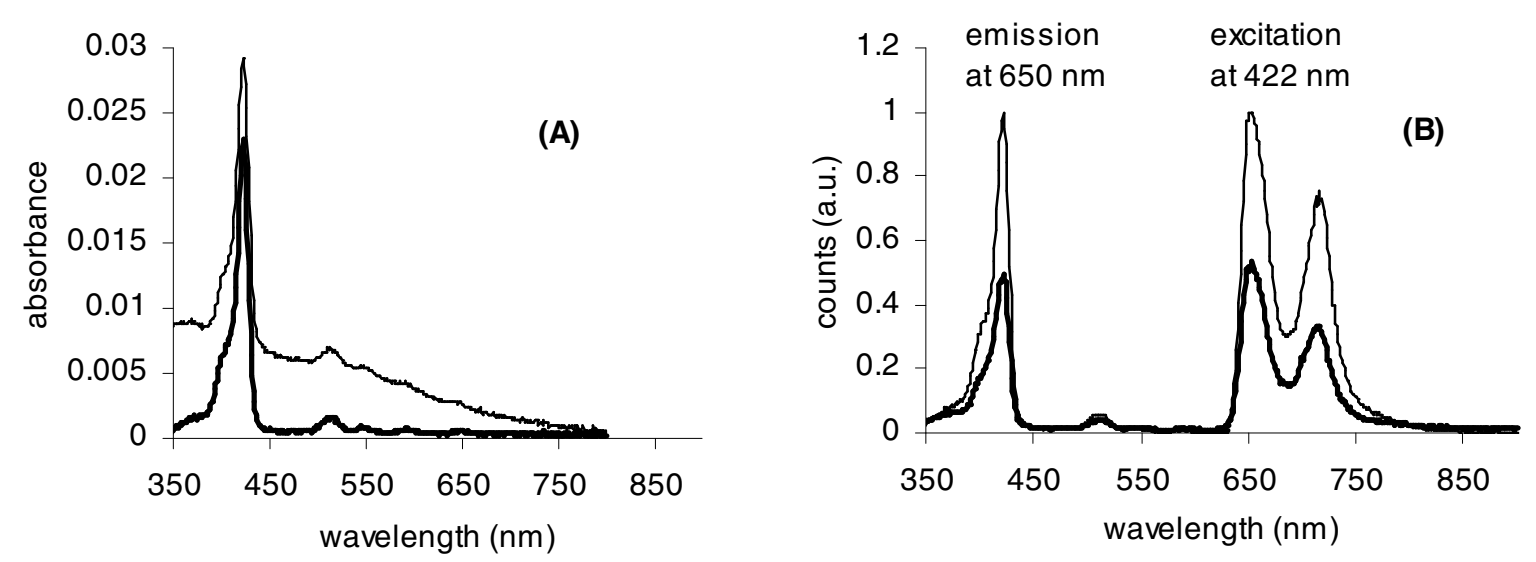

Figure S2. Absorption spectrum (A) and emission and excitation spectra (B) of P-ref (bold line) and P-reflAuNP (thin line) films. 


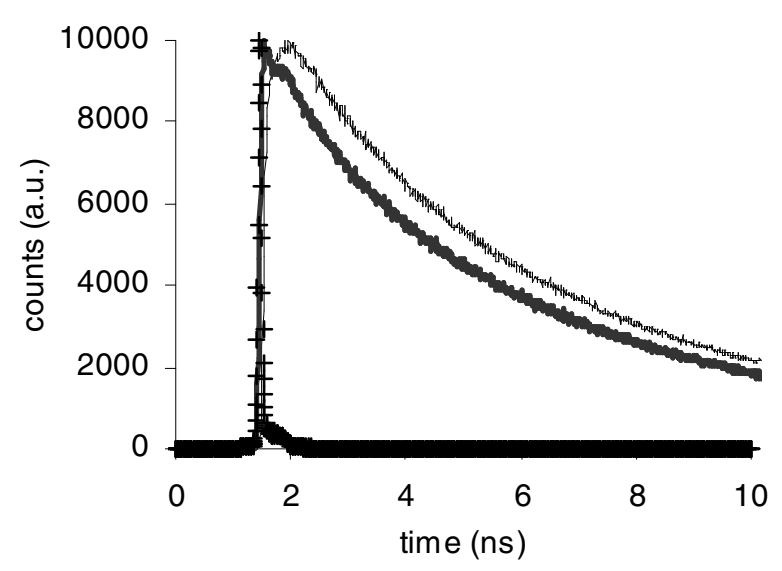

Figure S3. Time-resolved fluorescence of the P-ref (black line) and P-reflAuNP (bold gray line) films with lamp signal (crosses).

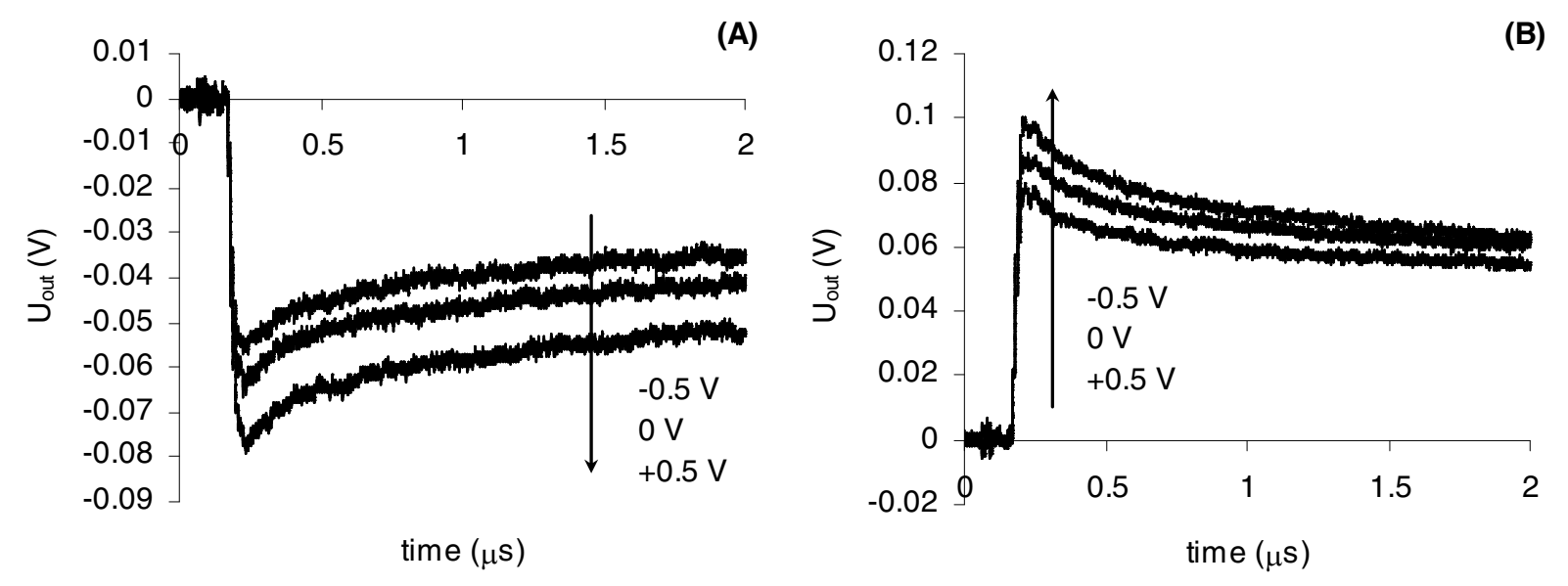

Figure S4. Effect of bias voltage variation from $-0.5 \mathrm{~V}$ to $+0.5 \mathrm{~V}$ on photovoltage of the structures (A) ITOIAuNPIPF; excitation energy density $0.028 \mathrm{~mJ} / \mathrm{cm}^{2}$ and (B) ITO|PF|AuNP; excitation energy density $0.52 \mathrm{~mJ} / \mathrm{cm}^{2}$. Excitation wavelength was $432 \mathrm{~nm}$.

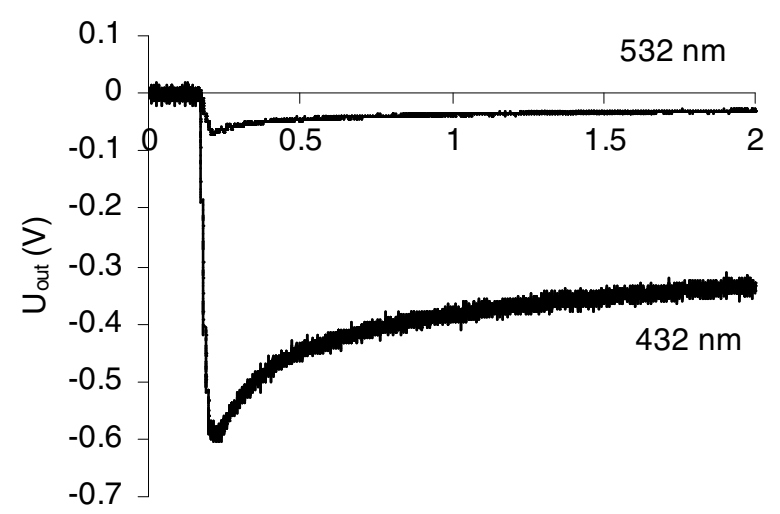

time $(\mu s)$

Figure S5. Photovoltage of structure ITOIAuNPIPF at wavelengths of 532 and $432 \mathrm{~nm}$ with a photon density of $1.1 \times 10^{15} \mathrm{~cm}^{-2}$ for both wavelengths. 